\title{
The acute effects of psyllium on postprandial lipaemia and thermogenesis in overweight and obese men
}

\author{
A. Khossousi, C. W. Binns, S. S. Dhaliwal and S. Pal* \\ Department of Nutrition, Dietetics and Food Science, ATN Centre for Metabolic Fitness, School of Public Health, \\ Curtin University, Perth, Western Australia 6102, Australia
}

(Received 7 March 2007 - Revised 21 August 2007 - Accepted 18 September 2007 - First published online 16 November 2007)

Overweight and obesity is one of the risk factors for developing CVD. At present, very little is known about the acute effects of dietary fibre on lipids, glucose and insulin, resting energy expenditure and diet-induced thermogenesis in overweight and obese individuals. This study examined the postprandial metabolic effects of dietary fibre in overweight and obese men. Ten overweight and obese men consumed a mixed meal accompanied by either a high-fibre or low-fibre supplement on two separate visits, in a random order, 1 week apart. Two isoenergetic breakfast meals with similar composition were consumed by ten overweight/obese men. The meals contained either a low ( $3 \mathrm{~g})$ or high ( $15 \mathrm{~g})$ amount of fibre, low-fibre meal (LFM) and high-fibre meal (HFM) respectively. Analysis was carried out using paired $t$ test and ANOVA. Serum TAG incremental area under the curve during $6 \mathrm{~h}$ of the postprandial period was significantly lower after the consumption of HFM compared with LFM. At the first hour of the postprandial period, plasma apo B48 concentration after consumption of HFM was significantly lower compared with LFM. The resting energy expenditure and diet-induced thermogenesis after both meals was similar during $6 \mathrm{~h}$ of the postprandial period. Collectively, these findings suggest that a single acute dose of dietary fibre in the form of psyllium supplement can decrease arterial exposure to TAG and modify chylomicron responses in the postprandial period.

Psyllium: Obesity: Postprandial state: Lipids: Lipoproteins: Thermogenesis

Overweight and obesity is one of the important risk factors in the development of CVD. Atherosclerosis is the main cause of CVD. Several lipoprotein types probably contribute to the initiation of atherosclerosis, including LDL and chylomicron remnants $(\mathrm{CMR})^{(1,2)}$. Under normal conditions, CMR are rapidly cleared from plasma predominantly via the LDLreceptor during the postprandial period. However, in the case of obesity, increased production and/or impaired clearance of these particles results in elevated plasma levels of postprandial chylomicrons and $\mathrm{CMR}^{(3)}$. Studies have also identified apo B48 as a marker of chylomicrons and CMR in femoral and carotid biopsies of human atherosclerotic plaque $^{(4-6)}$. Furthermore, studies have shown an increase in plasma CMR in subjects with or at risk of atherosclerosis ${ }^{(7,8)}$. As postprandial dyslipidaemia is an independent risk factor for atherosclerosis and $\mathrm{CVD}^{(8-11)}$, this phenomenon may be an additional cause of rapid progress of atherosclerosis among overweight and obese individuals.

Epidemiological studies have demonstrated that the prevalence of obesity is lower in populations that consume high-fibre diets compared with populations that consume low-fibre diets ${ }^{(12-14)}$. Similarly, within population groups, individuals with a higher fibre intake tend to be leaner than individuals with a lower fibre intake and case-control studies have documented that obese individuals typically consume less fibre than normal-weight individuals ${ }^{(14-16)}$. The main rationale for the effect of dietary fibre in weight control is based on the findings that dietary fibre may reduce food energy density and increase the satiating effect of a meal. However, an increase in the amount of dietary fibre in the diet may also influence resting energy expenditure (REE) and substrate utilisation due to altered postprandial lipid, glucose and insulin metabolism. Although the effect of dietary fibre on daily energy metabolism may be important in long-term weight control, this area has attracted little attention, especially in the overweight and obese ${ }^{(17,18)}$.

Dietary fibre is a collective term for a variety of plant substances that are resistant to digestion by human gastrointestinal enzymes ${ }^{(13,19)}$. Psyllium husk fibre (PSY) is a viscous, mostly water-soluble fibre and has been shown to be an effective supplement in adjunct to dietary intervention to control blood cholesterol and glucose levels ${ }^{(20)}$.

Most studies examining the effects of dietary fibre on postprandial lipaemia have been performed in healthy subjects and the results of studies examining the effect of different fibre types on postprandial lipid responses are variable ${ }^{(21)}$.

Studies demonstrating the beneficial effects of psyllium on hypercholesterolaemia have been done on hypercholesterolaemic or diabetic subjects. However, there is no information

Abbreviations: CMR, chylomicron remnant; DIT, diet-induced thermogenesis; HFM, high-fibre meal; IAUC, incremental area under the curve; LFM, low-fibre meal; PSY, psyllium husk fibre; REE, resting energy expenditure.

* Corresponding author: Professor Sebely Pal, fax +61 892662958 , email s.pal@curtin.edu.au 
available on the effect of PSY on the postprandial blood TAG and chylomicron responses in overweight and obese individuals. Therefore, the aim of the present study was to investigate the effect of PSY on postprandial blood biochemistry and dietinduced thermogenesis (DIT) in overweight and obese individuals.

\section{Experimental methods}

Subjects

Twelve overweight/obese male subjects, between the ages of 30 and 72 years, were recruited from the community. Subjects were screened for BMI $>25 \mathrm{~kg} / \mathrm{m}^{2}$ and waist circumference of $\geq 102 \mathrm{~cm}$. Exclusion criteria included smoking, lipid-lowering medication, use of medications that may influence lipid metabolism, such as steroids, warfarin, hypothyroidism, consumption of more than two standard alcoholic drinks per $\mathrm{d}$, diabetes mellitus, cardiovascular events within the last 6 months, major systemic diseases, gastrointestinal and gallbladder surgery, liver and renal diseases. The protocol was reviewed and approved by the Ethics Committee of Curtin University.

\section{Experimental protocol}

The present study was a randomised crossover design, which examined the effects of a dietary fibre supplement on postprandial lipaemia, glycaemia and insulin response after a high- or low-fibre standard meal (Table 1). The study included two separate intervention days, each proceeded by 1 week wash-out period. During the wash-out period subjects were required to follow their normal dietary habits and complete a food diary of their usual dietary intake $3 \mathrm{~d}$ before each intervention. Throughout the study period, subjects were asked to maintain their normal diet and refrain from vigorous physical activity. Subjects were supplied with a standard frozen meal to be consumed the night before intervention. On the test day, subjects arrived at Curtin University at 07.00 hours by car (to minimise physical activity) and after a $10-12 \mathrm{~h}$ fast. After subjects voided and were weighed, they rested in a supine position on a bed for $30 \mathrm{~min}$. Fasting blood samples

Table 1. Food content and composition of test meals*

\begin{tabular}{lcc}
\hline Ingredients & High-fibre meal & Low-fibre meal \\
\hline White bread (g) & 60 & 60 \\
Instant rice (g) & 25 & 25 \\
Whole milk (g) & 150 & 150 \\
Cream (g) & 20 & 20 \\
Cheese (g) & 20 & 20 \\
Sugar (g) & 13 & 13 \\
Skimmed milk (g) & 40 & 40 \\
Apple juice (g) & 200 & 200 \\
Psyllium husk (g) & 12 & - \\
Total energy from: (\%) & & 15 \\
$\quad$ Protein & 15 & 60 \\
$\quad$ Carbohydrate & 60 & 25 \\
$\quad$ Fat & 25 & 3 \\
Dietary fibre (g) & 15 & \\
\hline
\end{tabular}

${ }^{*}$ For details of subjects and procedures, see Experimental methods. were collected into EDTA vacutainer tubes, via an indwelling catheter into the antecubital vein. The test meal was then administered and blood samples were taken thereafter at hourly intervals for $4 \mathrm{~h}$ and then every $2 \mathrm{~h}$ for $6 \mathrm{~h}$.

\section{Test meal}

The two test meals were identical except for their fibre content. The high-fibre meal (HFM) had $12 \mathrm{~g}$ additional fibre in the form of psyllium husk supplement, which was consumed with $250 \mathrm{ml}$ water just before eating the test meal. The subjects consumed the same amount of water plus $5 \mathrm{~g}$ crushed instant rice instead of psyllium, as a placebo supplement, before eating the test meal on the other session. The composition of the standard meals is shown in Table 1 .

\section{Energy expenditure}

Energy expenditure was measured by indirect calorimetry using the Vmax-29 metabolic monitor (Sensor Medics) and open-circuit ventilated canopy measurement system. The measurement was conducted under standardised conditions with subjects lying (1) at complete physical rest; (2) in a thermally neutral environment; (3) with a minimum of $8 \mathrm{~h}$ of sleep; (4) awake and emotionally undisturbed; (5) without disease and fever. The measurements were taken based on the timetable explained earlier.

The Vmax-29 (Sensor Medics) was calibrated on the morning of each experimental day using a two-point calibration based on two separate mixtures of known gas content. Flow calibrations were achieved using a calibrated 3 litre syringe as directed in the manufacturer's instructions. Flow rate on each measurement day was set at 45 litres $/ \mathrm{min}$. On the morning of each experimental day, the instruments were re-calibrated for flow and the analysers for drift $2 \mathrm{~h}$ before consumption of the test meal. Performance of the instrument was also checked at regular intervals during the study period by monitoring the $\mathrm{CO}_{2}$ produced and $\mathrm{O}_{2}$ consumed for 30 min ethanol burns ${ }^{(22)}$.

\section{Hunger and satiety rating}

The subjects recorded their hunger feeling, fullness, desire to eat and prospective food consumption on a $100 \mathrm{~mm}$ visual analogue scale, without partition, at the left end 'not at all' and at the other end 'very much'. Visual analogue scale scores were recorded immediately before and every hour after the meal for $6 \mathrm{~h}^{(17,23)}$.

\section{Laboratory analysis}

Blood samples were centrifuged at approximately $2500 \mathrm{~g}$ at $4^{\circ} \mathrm{C}$ for $10 \mathrm{~min}$ and plasma was collected for determination of apo B48, plasma total cholesterol, HDL-cholesterol, TAG, insulin and glucose. Samples that were not immediately analysed were stored at $-80^{\circ} \mathrm{C}$ for later analysis.

Plasma TAG and total cholesterol were measured by enzymatic colorimetric kits (TRACE Scientific Ltd, Melbourne, Australia). Plasma HDL-cholesterol was measured after precipitation of apo B-containing lipoproteins with phosphotungstic acid and $\mathrm{MgCl}_{2}$; the supernatant containing 
the HDL-cholesterol was determined by enzymatic colorimetry (TRACE Scientific Ltd). Plasma LDL-cholesterol was determined by using a modified version of the Friedewald equation.

Apo B48 was quantified using a Western blotting/chemiluminescence procedure. Briefly, apolipoproteins were separated by SDS-PAGE using a Novex Mini-cell Electrophoresis system (Novex, San Diego, USA) with precast NUPAGE 3-8\% trisacetate gels according to the manufacturer's instructions. After electrophoresis, separated lipoproteins were transferred to a polyvinylidene fluoride membrane $\left(0.45 \mu \mathrm{m}\right.$; Immobilon $\mathrm{P}^{\mathrm{TM}}$; Milipore Corporation, Bedford, MA, USA). Apo B48 bands were identified and visualised using an antibody to apo B (DAKO A/S, Glostrup, Denmark) and subsequently anti-rabbit IgG antibody (HRP conjugated; Amersham Pharmacia Biotech, Amersham, UK) using enhanced chemiluminescence. Densitometric scanning of apo B48 bands and standardisation to known apo B48 protein mass allowed quantification of the protein. The mean intra- and inter-assay CV for apo B48 were each $<4 \%$.

Plasma insulin was measured by an ELISA kit (Dako Diagnostic, Japan). The homeostasis model assessment score was used as a surrogate estimate of the state of insulin sensitivity by multiplying fasting insulin concentration (mIU/l) with fasting glucose concentration (mmol/l) and dividing by 22.5 .

\section{Statistical analysis}

All results are given as means with their standard errors of the mean. Data were analysed for normality and responses to the two test meals were compared by ANOVA for repeated, paired measurement with time, diet and subjects as factors. Where the factors or the interactions were statistically significant $(P<0.05)$, a least significant difference test was used to determine whether the value at a given time and dietary treatment differed from $0 \mathrm{~h}$ (a significant postprandial response). Moreover, a paired $t$ test was used to determine significant differences between dietary treatments at a given time point. Incremental area under the curve (IAUC) was calculated for each measurement as the integrated area of the response and adjusted based on the baseline values. A paired $t$ test was used in the comparisons between two means. Statistical analysis was done using SPSS for windows (SPSS Inc., Chicago, IL, USA).
Table 2. Clinical characteristics of subjects at baseline† (Mean values with their standard errors)

\begin{tabular}{lclc}
\hline Characteristics* $^{*}$ & Mean & SEM & Range \\
\hline Age (years) & 57 & 4.5 & $30-72$ \\
Weight $(\mathrm{kg})$ & 91.1 & 1.93 & $72 \cdot 0-109.6$ \\
BMI $\left(\mathrm{kg} / \mathrm{m}^{2}\right)$ & 30.90 & 0.88 & $27 \cdot 1-37.0$ \\
Waist circumference $(\mathrm{cm})$ & 107.7 & 3.2 & $102-123$ \\
Waist:hip ratio & 0.98 & 0.12 & $0.90-1.04$ \\
HOMA score & 2.03 & 0.28 & $0.91-3.3$ \\
Resting energy expenditure $(\mathrm{kJ} / \mathrm{h})$ & 273 & 9 & $210-350$ \\
\hline
\end{tabular}

HOMA, homeostasis model assessment.

${ }^{*}$ Clinical characteristics of overweight/obese participants at screening.

†For details of subjects and procedures, see Experimental methods.

\section{Results}

\section{Subject characteristics}

Twelve subjects were initially recruited to the present study, with ten subjects completing the study. As assessed by their food records and subject interviews, subjects' diet and exercise habits were stable during the experimental period. The mean and range of clinical characteristics of subjects are shown in Table 2.

\section{Nutritional assessment}

Table 3 shows the mean $3 \mathrm{~d}$ dietary intake of subjects prior to each visit. There were no significant differences in energy, fibre and macronutrient intake, and no correlations were found between nutrient intake and lipid, lipoprotein and glucose postprandial responses.

\section{Postprandial lipids}

As shown in Fig. 1, blood TAG concentration increased following consumption of both a HFM and low-fibre meal (LFM). A peak in postprandial TAG concentrations was observed at $4 \mathrm{~h}$ for both meals. The blood TAG concentration was lower in the first and fourth hour following the HFM in comparison with the LFM (1.17 (SEM 0.03) compared with 1.32 (SEM 0.06 ) $\mathrm{mmol} / \mathrm{l}$ at the first hour, 1.54 (SEM 0.08) compared with 1.75 (SEM 0.06) $\mathrm{mmol} / \mathrm{l}$ at the fourth hour respectively).

Table 3. Nutritional assessment of food records $3 \mathrm{~d}$ prior to postprandial visit* (Mean values with their standard errors)

\begin{tabular}{lccccc}
\hline & \multicolumn{2}{c}{ Visit 1 } & & \multicolumn{2}{c}{ Visit 2 } \\
\cline { 2 - 3 } \cline { 5 - 6 } & Mean & SEM & & Mean & SEM \\
\hline Energy (kJ) & 8735.35 & 520.34 & & 8565 & 510.65 \\
Carbohydrate (\% total energy) & 51.33 & 2.2 & & 53.7 & 1.7 \\
Protein (\% total energy) & 16.54 & 0.79 & & 15.9 & 0.85 \\
Total fat (\% total energy) & 32.16 & 1.56 & & 30.63 & 1.89 \\
Saturated fat (\% total energy) & 14.66 & 1.02 & & 14.78 & 0.97 \\
Monounsaturated fat (\% total energy) & 13.79 & 1.07 & & 11.72 & 1.04 \\
Polyunsaturated fat (\% total energy) & 3.71 & 0.36 & & 3.93 & 0.42 \\
Dietary fibre (g) & 19.67 & 1.32 & & 20.36 & 0.96 \\
\hline
\end{tabular}

There was no significant difference in dietary intake at the start and finish of the intervention *For details of subjects and procedures, see Experimental methods. 


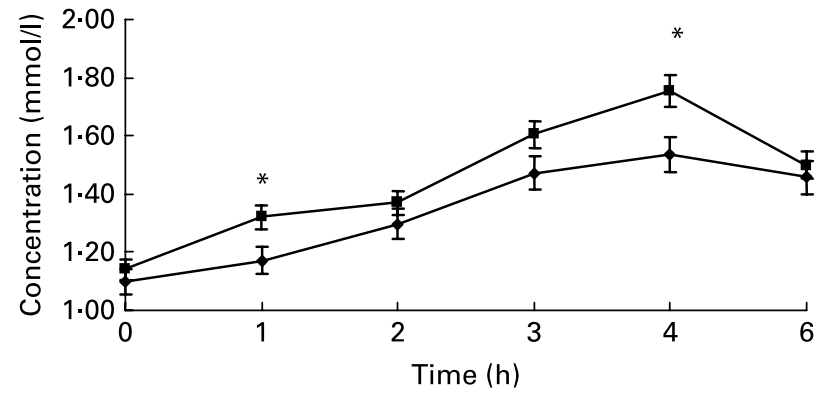

Fig. 1. Postprandial serum TAG concentrations over $6 \mathrm{~h}$ following consumption of a high-fibre meal $(--)$ ) or low-fibre meal $(-\mathbf{-}-)$. Values are means with their standard errors of the mean. Mean values were significantly different from controls: ${ }^{\star} P<0.05$. For details of subjects and procedures, see Experimental methods.

Moreover, consumption of the HFM produced a significant delay in the initial postprandial TAG response between 0 and $1 \mathrm{~h}$ relative to the LFM. The IAUC of TAG was significantly $21 \%$ lower following consumption of the HFM compared with the LFM within the $6 \mathrm{~h}$ studied. There was no change in total, LDL- or HDL-cholesterol following consumption of both meals during the $6 \mathrm{~h}$ postprandial period. The baseline and IAUC values of lipids are shown in Table 4.

\section{Postprandial apo B48 levels}

Apo B48 is an indicator of chylomicron and CMR metabolism and was used to monitor postprandial chylomicron/CMR changes. The plasma apo B48 concentration increased after consumption of both meals and reached the peak within $4 \mathrm{~h}$. As shown in Fig. 2, plasma apo B48 concentration was significantly lower after consumption of a HFM compared with a LFM at the first hour (10.04 (SEM 0.07) compared with 11.15 (SEM 0.04) $\mathrm{mg} / \mathrm{l}$ respectively). The baseline and IAUC values of apo B48 are shown in Table 4.

\section{Postprandial insulin and glucose levels}

Plasma glucose increased following consumption of both meals and postprandial glucose excursions were complete within $2-4 \mathrm{~h}$. Consumption of a HFM did not significantly affect the area under the curve and plasma concentrations for glucose and insulin compared with LFM within the $6 \mathrm{~h}$ studied. Therefore, consumption of a HFM did not improve glycaemic and insulinaemic responses in overweight/obese individuals who had some degree of insulin resistance. The baseline and IAUC values of glucose and insulin are shown in Table 4.

\section{Hunger and satiety}

There was no difference in the $6 \mathrm{~h}$ rating for hungry, satiety, desire to eat and prospective food consumption between the two tests (Fig. 3).

\section{Resting energy expenditure and respiratory quotient}

There were no significant differences in REE and RQ baseline values within the two sessions. REE increased significantly following both meals and returned to baseline within $6 \mathrm{~h}$. However, there was no difference in REE total area under the curve and DIT between meals during the $6 \mathrm{~h}$ postprandial period. The RQ levels were significantly lower after consumption of the HFM during the first $2 \mathrm{~h}$ compared with the LFM (0.91 (SEM 0.03) compared with 0.96 (SEM 0.06) nmol/1 at the first hour and 0.90 (SEM 0.04) compared with 0.95 (SEM $0.08) \mathrm{nmol} / \mathrm{l}$ at the second hour respectively). RQ IAUC was not different during the postprandial period between the HFM and LFM. The baseline and postprandial values of REE, RQ and DIT are shown in Table 5.

\section{Discussion}

The postprandial state directly reflects the process of digestion-assimilation of lipid and nutrients to which we are exposed most of the day. This state is characterised by rapid changes in blood TAG and chylomicron levels. The findings of the present study showed that a single dose of PSY supplementation was sufficient to decrease postprandial lipaemia and delay chylomicron production/secretion as measured by IAUC for TAG and apo B48 concentrations respectively.

Potential mechanisms by which psyllium may reduce fat digestion and absorption have been explained by Lairon and colleagues $^{(14,24)}$. Dietary fibre can change the postprandial responses by delaying gastric emptying and, thus, may also alter absorption of nutrients. Several studies have investigated

Table 4. Baseline value and postprandial incremental area under the curve (IAUC) for TAG, apo B48, total cholesterol, LDL-cholesterol, HDL-cholesterol, glucose and insulin*

(Mean values with their standard errors)

\begin{tabular}{|c|c|c|c|c|c|c|c|c|c|c|}
\hline & \multicolumn{2}{|c|}{ Baseline HFM } & \multicolumn{2}{|c|}{ Baseline LFM } & \multirow[b]{2}{*}{$P$ value } & \multicolumn{2}{|c|}{ IAUC HFM } & \multicolumn{2}{|c|}{ IAUC LFM } & \multirow[b]{2}{*}{$P$ value } \\
\hline & Mean & SEM & Mean & SEM & & Mean & SEM & Mean & SEM & \\
\hline Apo B48 (mg/l) & $8 \cdot 81$ & 0.37 & $9 \cdot 91$ & 0.55 & 0.874 & $19 \cdot 20$ & $6 \cdot 19$ & $21 \cdot 21$ & 4.94 & 0.803 \\
\hline Total cholesterol $(\mathrm{mmol} / \mathrm{l})$ & $4 \cdot 76$ & 0.27 & 4.97 & 0.23 & 0.970 & $29 \cdot 70$ & 1.55 & $30 \cdot 28$ & $1 \cdot 80$ & 0.711 \\
\hline LDL-cholesterol (mmol/l) & $2 \cdot 87$ & 0.28 & 2.93 & 0.32 & 0.928 & $16 \cdot 60$ & 1.89 & $16 \cdot 92$ & $2 \cdot 11$ & 0.928 \\
\hline HDL-cholesterol (mmol/l) & $1 \cdot 18$ & 0.08 & $1 \cdot 15$ & 0.09 & 0.893 & $7 \cdot 83$ & 0.71 & 7.45 & 0.61 & 0.584 \\
\hline Insulin $(\mu \mathrm{IU} / \mathrm{ml})$ & $7 \cdot 68$ & $1 \cdot 18$ & $7 \cdot 38$ & $1 \cdot 10$ & 0.885 & 62.57 & $15 \cdot 77$ & 67.73 & 14.43 & 0.855 \\
\hline
\end{tabular}

HFM, high-fibre meal; LFM, low-fibre meal.

${ }^{*}$ For details of subjects and procedures, see Experimental methods. 


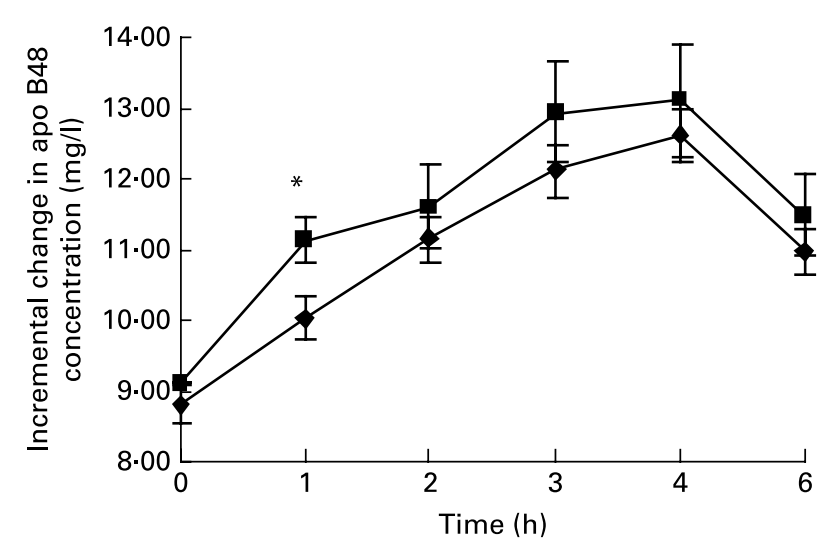

Fig. 2. Postprandial serum apo B48 concentrations over $6 \mathrm{~h}$ following consumption of a high-fibre meal $(--)$ or low-fibre meal $(-\mathbf{-}-)$. Data are means with their standard errors of the mean. Mean values were significantly different from controls: ${ }^{\star} P<0.05$. For details of subjects and procedures, see Experimental methods. the effect of dietary fibre in gastric emptying of different meals ${ }^{(23,25-27)}$ and shown that soluble dietary fibre could delay the gastric emptying of the liquid phase (water, energy-containing liquids such as glucose or amino acids solutions), but a specific effect on the solid phase, which contains lipids, has not been reported ${ }^{(28)}$.

It has been suggested that some dietary fibres can alter pancreatic lipase activity. Lairon et al. ${ }^{(29)}$ demonstrated that when pancreatic lipase is mixed with high-fibre gastric contents and bile in the duodenum, some entrapment of pancreatic lipase occurs; it was reported in in vitro conditions mimicking the physiological conditions. In gastric conditions, soluble fibres that produced sufficient viscosity in solution significantly lower the extent of lipid emulsification and, consequently, reduced the extent of TAG hydrolysis catalysed by gastric lipase. In duodenal conditions, the extent of emulsification was negatively correlated to the viscosity of fibre ${ }^{(21,30,31)}$. Thus, these findings indicate that reducing emulsification of

(a) 80 How hungry do you feel?
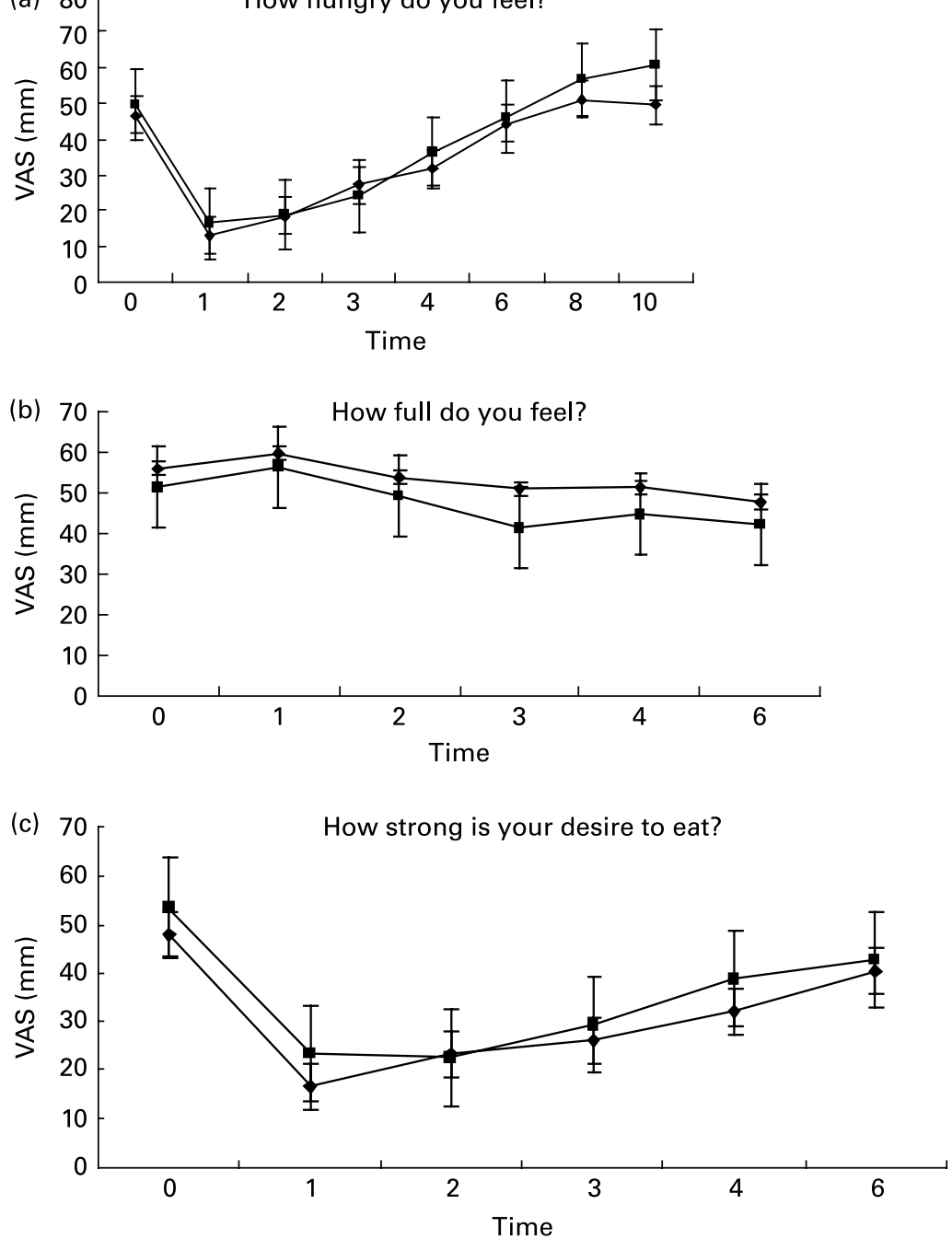

Fig. 3. Postprandial hunger (a), satiety (b) and desire to eat (c) rating responses. There was no difference in the hunger, satiety, desire to eat and prospective food consumption. VAS, visual analogue scale. For details of subjects and procedures, see Experimental methods. 
Table 5. The influence of the high-fibre meal (HFM) on postprandial thermogenesis and RQ over $6 \mathrm{~h}$ in overweight and obese men*

(Mean values with their standard errors)

\begin{tabular}{|c|c|c|c|c|c|}
\hline & \multicolumn{2}{|c|}{ HFM } & \multicolumn{2}{|c|}{ LFM } & \multirow[b]{2}{*}{$P$ value } \\
\hline & Mean & SEM & Mean & SEM & \\
\hline \multicolumn{6}{|l|}{ Fasting } \\
\hline REE (kJ/h) & 271 & 11 & 276 & 13 & 0.493 \\
\hline $\mathrm{RQ}$ & 0.89 & 0.01 & 0.90 & 0.01 & 0.347 \\
\hline \multicolumn{6}{|l|}{ Postprandial } \\
\hline DIT (kJ/6h) & 174 & 23 & 187 & 35 & 0.709 \\
\hline DIT \% energy intake & $5 \cdot 4$ & 0.7 & $5 \cdot 8$ & $1 \cdot 1$ & 0.709 \\
\hline RQ IAUC & 0.19 & 0.06 & 0.19 & 0.03 & 0.976 \\
\hline
\end{tabular}

LFM, low-fibre meal; REE, resting energy expenditure; DIT, diet-induced thermogenesis; IAUC, incremental area under the curve.

${ }^{*}$ For details of subjects and procedures, see Experimental methods.

dietary lipids in the stomach and the duodenum is a mechanism by which soluble viscous fibres can alter lipid assimilation. Moreover, viscous fibres may increase the thickness of the unstirred water layer, which can also decrease nutrient absorption rate ${ }^{(32)}$. These effects likely explain the reduced rates of diffusion and absorption of lipids as examined in acute studies in the presence of highly viscous fibres ${ }^{(33)}$.

Indeed, a few postprandial studies investigated the acute effects of dietary fibre on postprandial lipidaemia in healthy subjects. In one study, adding $10 \mathrm{~g}$ wheat fibre significantly $(-20 \%)$ reduced the TAG area under the curve postprandially ${ }^{(34)}$. In another study, adding $10 \mathrm{~g}$ oat bran to a test meal led to a $37 \%$ reduction of postprandial TAG responses ${ }^{(35)}$. The results of the current study show that psyllium husk can decrease TAG concentration and modify chylomicron responses in circulation postprandially, probably by altering lipid processing and reducing the rates of diffusion and absorption of lipids ${ }^{(28)}$.

Postprandial glucose and insulin response after consumption of a HFM was not significantly different compared with a LFM and there was only a delayed peak of plasma glucose level after consumption of the HFM compared with the LFM (data not shown). Previous studies have demonstrated that dietary fibre consumption, including psyllium, is associated with improved postprandial glucose response and insulin resistance ${ }^{(36,37)}$. However, these effects are predominantly observed in type II diabetics and with chronic consumption of psyllium. Other studies show variable effects of psyllium on blood glucose responses in normal and diabetic subjects ${ }^{(38-41)}$. The inconsistent results may be attributed to how psyllium is administrated and partly to the physical properties of the psyllium. Wolver showed that when psyllium was consumed in the form of a supplement it did not reduce plasma glucose levels in the postprandial phase and mixing the fibre with the meal may be necessary to modify the postprandial glucose and insulin responses ${ }^{42)}$. In studies where viscous fibre supplements were taken in water before meals or with snacks and sweets taken after or separately from major meals ${ }^{(43,44)}$, there was no benefit on blood glucose control. It might be explained by the effect of psyllium husk on gastric emptying. As mentioned earlier, psyllium can alter the liquid phase of gastric emptying, including water-soluble nutrients such as glucose ${ }^{(21)}$. Therefore, the initial delay in the beginning of postprandial glucose excursion could be explained by alteration in liquid phase gastric emptying.
DIT is the result of energy extended to digest, transport, metabolise and store food. It averages about $10-15 \%$ total daily energy expenditure and varies with the metabolic fate of the ingested substrate ${ }^{(45)}$. Studies have demonstrated a decrease in DIT after consumption of a $\operatorname{HFM}^{(17,18)}$. Scalfi et al. showed a reduction in $6 \mathrm{~h}$ DIT after adding $6 \mathrm{~g}$ glucomannan (soluble fibre) or $26 \mathrm{~g}$ dietary fibre to the meal in seven healthy subjects. Raben et al. found that an additional intake of $3.5 \mathrm{~g}$ pea fibre decreased the 6-h DIT and fat oxidation rate in ten normal-weight subjects. There is evidence to suggest that obese individuals may have a defective thermogenic response to meal ingestion when compared with lean individuals ${ }^{(46)}$. De Jonge and Bray concluded that DIT was lower in obesity, an outcome demonstrated in twenty-two of twenty-nine studies examined ${ }^{(47)}$. This difference in thermogenesis was accompanied by significant changes in both carbohydrate and fat oxidation. All the participants in the present study were overweight or obese; therefore, the reason for no changes in DIT and substrate oxidation between groups may be because of the suppressed DIT in this particular group. As shown before, the DIT percentage of food intake after the meal without fibre supplement was about 5, which represents a blunted metabolic response to food intake. Therefore, the fibre consumption in this group does not show a negative effect on DIT and fat oxidation.

Dietary fibre can alter energy intake by different mechanisms. A fibre-enriched meal is processed more slowly and nutrient absorption occurs over a longer period ${ }^{(48)}$. Furthermore, dietary fibre affects gastric distension, the rate of gastric emptying and intestinal transit time ${ }^{(49)}$. Many studies demonstrated that dietary fibre could change energy intake without an effect on hunger and satiety rate. Pasman et al. studied the effect of 1 week of fibre supplementation in the form of soluble fibre on hunger, satiety and energy intake ${ }^{(50)}$. With consumption of $40 \mathrm{~g}$ guar gum per $\mathrm{d}$, mean daily energy intake decreased significantly from $6.7 \mathrm{MJ}$ to $5.4 \mathrm{MJ}$, whereas hunger and satiety scores did not change. Heini et al. showed that consumption of soluble fibre in the form of hydrolysed guar did not change the satiety rate compared with a placebo ${ }^{(51)}$. In the present study, the hunger and satiety rates were not different after consumption of HFM compared with LFM in the short term. Therefore, further investigation is necessary to examine the effect of long-term consumption of fibre supplement on energy intake and weight control.

In conclusion, we have shown that consumption of a HFM containing dietary fibre as a single supplement, containing $12 \mathrm{~g}$ psyllium husk, had immediate beneficial effects on postprandial TAG and chylomicron concentrations in overweight/ obese men compared with a LFM. Studies have shown that postprandial glycaemic and insulinaemic responses have strong effects on TAG and chylomicron production and clearance ${ }^{(28,52)}$. Interestingly, the postprandial glycaemic and insulinaemic responses did not improve after consumption of the HFM compared with the LFM. Therefore, the postprandial lipid alterations, which were observed following consumption of the HFM, may be independent of glycaemic and insulinaemic responses and could be due to changes in digestion and probably absorption rate of lipids. Collectively, these findings suggest a single dose of dietary fibre in the form of a PSY supplement can decrease arterial exposure to TAG and modify chylomicron responses in the postprandial period. 


\section{References}

1. Karpe F (1999) Postprandial lipoprotein metabolism and atherosclerosis. J Intern Med 246, 341-355.

2. Alipour A, Elte JW, van Zaanen HC, Rietveld AP \& Cabezas MC (2007) Postprandial inflammation and endothelial dysfunction. Biochem Soc Trans 35, Pt 3, 466-469.

3. Austin MA, Goto Y, Lenfant C \& Tyroler HA (1991) The hypertriglyceridemias: risk and management. Am $J$ Cardiol 68, 22A-25A.

4. Yu KC, Smith D, Yamamoto A, et al. (1997) Phagocytic degradation of chylomicron remnants by fibroblasts from subjects with homozygous familial hypercholesterolaemia. Clin Sci (Lond) 92, 197-203.

5. Yu KC \& Mamo JC (2000) Chylomicron-remnant-induced foam cell formation and cytotoxicity: a possible mechanism of cell death in atherosclerosis. Clin Sci (Lond) 98, 183-192.

6. Mamo JC, Elsegood CL, Gennat HC \& Yu K (1996) Degradation of chylomicron remnants by macrophages occurs via phagocytosis. Biochemistry 35, 10210-10214.

7. Simons LA, Dwyer T, Simons J, et al. (1987) Chylomicrons and chylomicron remnants in coronary artery disease: a case-control study. Atherosclerosis 65, 181-189.

8. Tanaka A (2004) Postprandial hyperlipidemia and atherosclerosis. $J$ Atheroscler Thromb 11, 322-329.

9. Mamo JC, Yu KC, Elsegood CL, et al. (1997) Is atherosclerosis exclusively a postprandial phenomenon? Clin Exp Pharmacol Physiol 24, 288-293.

10. Proctor SD \& Mamo JC (1996) Arterial fatty lesions have increased uptake of chylomicron remnants but not low-density lipoproteins. Coron Artery Dis 7, 239-245.

11. Karpe F, Steiner G, Uffelman K, Olivecrona $T$ \& Hamsten A (1994) Postprandial lipoproteins and progression of coronary atherosclerosis. Atherosclerosis 106, 83-97.

12. Van Itallie TB (1978) Dietary fiber and obesity. Am J Clin Nutr 31, 10 Suppl., S43-S52.

13. Howarth NC, Saltzman E \& Roberts SB (2001) Dietary fiber and weight regulation. Nutr Rev 59, 129-139.

14. Lairon D (2007) Dietary fiber and control of body weight. Nutr Metab Cardiovasc Dis 17, 1-5.

15. Alfieri MA, Pomerleau J, Grace DM \& Anderson L (1995) Fiber intake of normal weight, moderately obese and severely obese subjects. Obes Res 3, 541-547.

16. Slavin JL (2005) Dietary fiber and body weight. Nutrition 21, 411-418.

17. Raben A, Christensen NJ, Madsen J, Holst JJ \& Astrup A (1994) Decreased postprandial thermogenesis and fat oxidation but increased fullness after a high-fiber meal compared with a low-fiber meal. Am J Clin Nutr 59, 1386-1394.

18. Scalfi L, Coltorti A, D'Arrigo E, et al. (1987) Effect of dietary fibre on postprandial thermogenesis. Int J Obes 11, Suppl. 1, 95-99.

19. Eastwood MA (1983) Dietary fiber. Lancet 2, 202-206.

20. Anderson JW (1987) Dietary fiber, lipids and atherosclerosis. Am J Cardiol 60, 17G-22G.

21. Lairon D (1996) Dietary fibres: effects on lipid metabolism and mechanisms of action. Eur J Clin Nutr 50, 125-133.

22. Soares MJ, Cummings SJ, Mamo JC, Kenrick M \& Piers LS (2004) The acute effects of olive oil $v$. cream on postprandial thermogenesis and substrate oxidation in postmenopausal women. Br J Nutr 91, 245-252.

23. Rigaud D, Paycha F, Meulemans A, Merrouche M \& Mignon M (1998) Effect of psyllium on gastric emptying, hunger feeling and food intake in normal volunteers: a double blind study. Eur J Clin Nutr 52, 239-245.

24. Lairon D (1996) Lipid absorption and metabolism: physiological and molecular aspects. Proc Nutr Soc 55, 1B, 1-3.
25. Ralphs DN, Thomson JP, Haynes S, Lawson-Smith C, Hobsley M \& Le Quesne LP (1978) The relationship between the rate of gastric emptying and the dumping syndrome. Br J Surg 65, 637-641.

26. Blackburn NA, Holgate AM \& Read NW (1984) Does guar gum improve postprandial hyperglycaemia in humans by reducing small intestinal contact area? Br J Nutr 52, 197-204.

27. Ryttig KR, Tellnes G, Haegh L, Boe E \& Fagerthun H (1989) A dietary fibre supplement and weight maintenance after weight reduction: a randomized, double-blind, placebo-controlled long-term trial. Int J Obes 13, 165-171.

28. Lairon D, Play B \& Jourdheuil-Rahmani D (2007) Digestible and indigestible carbohydrates: interactions with postprandial lipid metabolism. J Nutr Biochem 18, 217-227.

29. Lairon D, Lafont H, Vigne JL, Nalbone G, Leonardi J \& Hauton JC (1985) Effects of dietary fibers and cholestyramine on the activity of pancreatic lipase in vitro. Am J Clin Nutr 42, 629-638.

30. Pastors JG, Blaisdell PW, Balm TK, Asplin CM \& Pohl SL (1991) Psyllium fiber reduces rise in postprandial glucose and insulin concentrations in patients with non-insulin-dependent diabetes. Am J Clin Nutr 53, 1431-1435.

31. Pasquier B, Armand M, Castelain C, et al. (1996) Emulsification and lipolysis of triacylglycerols are altered by viscous soluble dietary fibres in acidic gastric medium in vitro. Biochem $J$ 314, Pt 1, 269-275.

32. Flourie B, Vidon N, Florent CH \& Bernier JJ (1984) Effect of pectin on jejunal glucose absorption and unstirred layer thickness in normal man. Gut 25, 936-941.

33. Gee JM, Blackburn NA \& Johnson IT (1983) The influence of guar gum on intestinal cholesterol transport in the rat. Br J Nutr 50, 215-224.

34. Cara L, Dubois C, Borel P, et al. (1992) Effects of oat bran, rice bran, wheat fiber, and wheat germ on postprandial lipemia in healthy adults. Am J Clin Nutr 55, 81-88.

35. Lia A, Andersson H, Mekki N, Juhel C, Senft M \& Lairon D (1997) Postprandial lipemia in relation to sterol and fat excretion in ileostomy subjects given oat-bran and wheat test meals. Am J Clin Nutr 66, 357-365.

36. Sierra M, Garcia JJ, Fernandez N, Diez MJ, Calle AP \& Sahagun AM (2001) Effects of ispaghula husk and guar gum on postprandial glucose and insulin concentrations in healthy subjects. Eur J Clin Nutr 55, 235-243.

37. Anderson JW, Allgood LD, Turner J, Oeltgen PR \& Daggy BP (1999) Effects of psyllium on glucose and serum lipid responses in men with type 2 diabetes and hypercholesterolemia. Am J Clin Nutr 70, 466-473.

38. Jarjis HA, Blackburn NA, Redfern JS \& Read NW (1984) The effect of ispaghula (Fybogel and Metamucil) and guar gum on glucose tolerance in man. Br J Nutr 51, 371-378.

39. Sartor G, Carlstrom S \& Schersten B (1981) Dietary supplementation of fibre (Lunelax) as a mean to reduce postprandial glucose in diabetics. Acta Med Scand Suppl 656, 51-53.

40. Asp NG, Agardh CD, Ahren B, et al. (1981) Dietary fibre in type II diabetes. Acta Med Scand Suppl 656, 47-50.

41. Florholmen J, Arvidsson-Lenner R, Jorde R \& Burhol PG (1982) The effect of Metamucil on postprandial blood glucose and plasma gastric inhibitory peptide in insulin-dependent diabetics. Acta Med Scand 212, 237-239.

42. Wolever TM, Vuksan V, Eshuis H, et al. (1991) Effect of method of administration of psyllium on glycemic response and carbohydrate digestibility. J Am Coll Nutr 10, 364-371.

43. Jenkins DJ, Wolever TM, Leeds AR, et al. (1978) Dietary fibres, fibre analogues, and glucose tolerance: importance of viscosity. Br Med J 1, 6124, 1392-1394.

44. Jenkins DJ, Jenkins AL, Wolever TM, Rao AV \& Thompson LU (1986) Fiber and starchy foods: gut function and implications in disease. Am J Gastroenterol 81, 920-930. 
45. Segal KR (1983) Thermic effects of food and exercise in lean and obese women. Metabolism 32, 581-589.

46. Raben A, Andersen HB, Christensen NJ, Madsen J, Holst JJ \& Astrup A (1994) Evidence for an abnormal postprandial response to a high-fat meal in women predisposed to obesity. Am J Physiol 267, 4 Pt 1, E549-E559.

47. de Jonge L \& Bray GA (1997) The thermic effect of food and obesity: a critical review. Obes Res 5, 622-631.

48. Jenkins DJ, Wolever TM, Rao AV, et al. (1993) Effect on blood lipids of very high intakes of fiber in diets low in saturated fat and cholesterol. $N$ Engl J Med 329, 21-26.

49. Kritchevsky D, Bonfield C \& Anderson JW (1990) Dietary Fiber: Chemistry, Physiology, and Health Effects. New York and London: Plenum Press.
50. Pasman WJ, Saris WH, Wauters MA \& Westerterp-Plantenga MS (1997) Effect of one week of fibre supplementation on hunger and satiety ratings and energy intake. Appetite 29, $77-87$.

51. Heini AF, Lara-Castro C, Schneider H, Kirk KA, Considine RV \& Weinsier RL (1998) Effect of hydrolyzed guar fiber on fasting and postprandial satiety and satiety hormones: a double-blind, placebo-controlled trial during controlled weight loss. Int J Obes Relat Metab Disord 22, 906-909.

52. Harbis A, Perdreau S, Vincent-Baudry S, et al. (2004) Glycemic and insulinemic meal responses modulate postprandial hepatic and intestinal lipoprotein accumulation in obese, insulin-resistant subjects. Am J Clin Nutr 80, 896-902. 